\title{
PENSAMIENTO ESTRATÉGICO BASE DEL MARKETING INTERNACIONAL EN EL SECTOR LÁCTEO
}

\author{
Nair Cantillo Campo Carlos Pedraza Reyes ${ }^{2}$
}

\section{Resumen}

El presente artículo tuvo por objeto analizar el pensamiento estratégico como base del marketing internacional en el sector de lácteo. El tipo de investigación fue descriptiva y documental, apoyado en un diseño bibliográfico, donde además se recurrió a una técnica de captación de información de igual naturaleza metodológica. En líneas generales se concluye que producto de la revisión documental se destaca que las empresas lácteas colombianas cuenta con capacidad para dar respuestas eficaces y eficientes a los clientes que integran su mercado en cuanto a infraestructura, tecnología, capital económico y talento humano para exportar sus productos, permitiendo satisfacer las necesidades sociales propias y de otros países, como es el caso Venezolano donde como consecuencia de ser fronterizo con Colombia se perciben con receptividad en los diversos sectores que integran su estructura social, sin embargo, se notan debilidades en este aspecto, por parte de las empresas del sector en estudio atribuido a factores como : carencia en la distribución y publicidad ,asimismo no tienen un servicio de post-venta, los productos en ocasiones escasean en los anaqueles, falta de servicio personalizado en los grandes supermercados para dar a conocerlos y los beneficios derivados de ellos entre otros.

Palabras claves: Pensamiento estratégico, marketing internacional necesidades sociales.

${ }^{1}$ Lcdo. Profesional en Relaciones internacionales. Especialización en Educación en Derechos Humanos.

Mg. Gerencia de Mercadeo. Aspirante a Dr. En Ciencias Gerenciales. Email: carlodjpedrazar@hotmail.com.

Teléfonos 300-8085672-317-7491680

${ }^{2}$ Lcda. Administración de empresas. Mg. Gerencia de Mercadeo. Dr. En Ciencias Gerenciales.

Email: nair.cantillo@unad.edu.co. Teléfono: 3015258139 


\section{STRATEGIC THINKING BASIS OF INTERNATIONAL MARKETING IN THE DAIRY SECTOR}

\section{Abstract}

This article was to analyze the strategic thinking as the basis of international marketing in the dairy sector. For this purpose texts were consulted as Martinez (1998). The research was descriptive and documentary, supported by a design literature, which also used a technique of gathering information of the same kind of methodology. Overall it is concluded that the document review product highlights that Colombian dairy companies has capacity for effective and efficient responses to customers that make up your market in terms of infrastructure, technology, economic capital and human talent to export their products allowing social needs their own and other countries, such as Venezuela, where as a result of being perceived borders Colombia to receptivity in the various sectors that make up the social structure, however, noticeable weaknesses in this aspect, part of the studied companies attributed to factors such as lack of distribution and advertising, also have no after-sales service, sometimes scarce products on the shelves, lack of personal service in large supermarkets to give know them and the benefits derived from them and others.

Keywords: Strategic thinking, international marketing social needs.

\section{Introducción}

La globalización de los mercados ha originado en las empresas nacionales la necesidad de expandirse para empezar a ser protagonistas en el ámbito internacional, mediante la consecución de nuevos clientes o socios que harán posible que ellas se vuelvan rentables y lograr mantenerse en el medio, a través de estrategias que generen ventajas competitivas.

A tal efecto, dentro de esta investigación analizaremos las estrategias de marketing internacional de las empresas del sector lácteo en Colombia, al igual que su situación actual para llegar a nuevos mercados, estas metas de proyección en los mercados internacionales serán factibles mediante el desarrollo del pensamiento estratégico para la propuesta de nuevas acciones de mercado, buscando apoyarse en un personal creativo e innovador, a fin de aperturar o ampliar la inserción de los productos o servicios ofertados en otros mercados. Pues bien, el factor fundamental tanto de los empresarios como de los gerentes hoy día es plantearse esquemas atractivos que faciliten no solo la supervivencia de las empresas en términos de rentabilidad sino también mantenerse competitiva, y ser socialmente responsable con su entorno, bien sea desde el campo de atención social como ambiental.

Por otro lado, perciben como que tanto a nivel nacional e internacional de este rubro de la economía está siendo amenaza por factores endógenos como son la inestabilidad económica, las políticas emitidas por el gobierno, la poca producción lechera, y el alto costo de los insumos, donde se ven afectada su estabilidad y permanencia. Por ello, las organizaciones que tienen como razón social la comercialización de bienes de consumo, en particular el sector lechero-lácteo, debe realizar esfuerzos, para proyectar en forma acertada sus productos, a través de me- 
dios de comunicación, asimismo utilizar otras herramientas apropiadas de manera que los lleve a ser competitivos y exitosos.

Siguiendo las pautas anteriores en este estudio se analizó el pensamiento estratégico como base para el marketing internacional en el sector lácteo.

\section{El pensamiento estratégico y el marketing internacional alianzas para un desarrollo sostenible}

En este sentido, vale destacar que a nivel internacional el comercio es favorable para las empresas que se encuentran preparadas para garantizar la satisfacción del cliente tanto interno como externo y cuando a su vez cuentan con elementos tangibles e intangibles como son la producción, infraestructura, transporte, sin dejar de lado al hombre como ente propulsor de ideas, entre otros elementos que dan vida activa al negocio y lo interrelaciona con el entorno, sin embargo los resultados se verán reflejados en ventajas competitivas para distinguirse entre otras del mismo rubro o similar.

Al respecto, mediante el discernimiento documental se destaca el análisis teórico donde se pretende dar una visión general sobre el tema objeto de estudio, y por otra parte conceptualizar las variables a las que se hace referencia. Para ello se inicia describiendo los principios que se formulan por autores, a fin de visualizar la importancia que tiene en el acontecer económico en general y las organizaciones en particular las del sector lácteo.

\section{Industria láctea fuente de crecimiento economico}

Es un sector de la industria que tiene como materia prima la leche procedente de los animales especialmente la vaca, siendo este producto uno de los elementos de la cesta básica alimentaria, los subproductos que genera esta indus- tria se categorizan como lácteos e incluyen existe una amplia gama que van desde los productos fermentados como el yogurt, queso, pasando por los no fermentados como las mantequillas y sueros. Dentro de este contexto los lácteos son productos perecederos que deben mantener rigurosamente la cadena de frío, es por esta razón que los envases están especialmente diseñados para salvaguardarlos, además ricos en contenido proteínico, grasos y carbohidratos.

Las características físicas y químicas de los lácteos se testean en muchos casos de forma similar a la leche, es decir, se emplean por ejemplo lactómetros para medir la densidad específica. No obstante la elaboración de los lácteos es diferente según el proceso que se haya realizado; por ejemplo algunos de ellos se han sometido a fermentación láctica (un ejemplo los yogures), otros por el contrario sufren un proceso mecánico de concentración de su contenido graso (mantequillas). A veces es posible un proceso combinado de fermentación y maduración (quesos).

Estos procesos cambian la composición y la concentración inicial de ciertos micronutrientes, dependiendo del lácteo en cuestión, la industria láctea tiene como primera restricción manipular la leche por debajo de los 7 grados centígrados.

Sobre el particular, del objeto de estudio los elementos en común que manejan tanto Colombia como Venezuela, representa el tipo de productos que se consumen, en el cual para ambos países constituye un producto de primera necesidad catalogado como esencial en la cesta básica, asimismo sus derivados, caso este la leche en el estado líquido, en polvo, los derivados como el yogur de frutas, los quesos duros, blandos, tajados, de untar, los sueros, cremas de leche, esto se debe a que se tienen una cultura similar donde se proyecta con costumbres y hábitos alimentarios en común, quizás derivados por la aproximada geográfica de las fronteras 


\section{Desarrollo del sector lácteo en el mercado doméstico e internacional}

Colombia es un país agrícola y ganadero por tradición, esto conlleva a tener un número considerable de procesadoras de leche y sus derivados, para la realización del trabajo se tomara como punto de referencia una empresa que tiene vínculos comerciales con Venezuela hace varios años y que paso de ser una empresa exportadora a tener sus propias plantas de producción en este país se está hablando de Alpina s.a, sus políticas de comercialización han sido tan exitosas que paso hacer parte de la industria venezolana, información que se encuentra disponible en www.alpina.com.col, y la segunda es la empresa Colanta Ltda, de la cual hace referencia esta investigación, puesto que está buscando entrar al mercado internacional. La Cooperativa Colanta es una empresa sin ánimo de lucro; conformada por más de 11.000 asociados productores y trabajadores. Recibe y procesa más de un millón de litros de leche diarios, parte es para producir leche líquida, otra para derivados y quesos blandos como de maduración.

Gracias a los controles de calidad en la leche, desde la ubre de las vacas hasta el consumidor final, los productos Colanta son los mejores y a los más bajos precios del mercado colombiano. Además, produce y comercializa cárnicos de muy alta calidad, se elabora concentrado para animales, sales mineralizadas y fertilizantes con un riguroso control de calidad. La Cooperativa Colanta es una claro ejemplo, de lo que se puede lograr con el sistema cooperativo y con la solidaridad de sus 11.000 proveedores de leche, que la han convertido en la pasteurizadora, comercializadora y distribuidora de leche más importante en Colombia, según el informe de Agrocadenas del Ministerio de Agricultura, Colanta ocupa el primer lugar de mayor venta de leche en Colombia.

COLANTA alcanzo ventas totales de un billón noventa y nueve mil trescientos treinta y cuatro millones de pesos (\$1.099.334.000.000.00) a 31 de diciembre de 2006 y excedentes superiores a $\$ 20$. Mil millones, como se describe en el balance. Informe de Gestión Colanta 2006. En la actualidad la organización cuenta con plantas procesadoras en diferentes partes del país, en los actuales momentos esta posesionada entre las 35 empresas más grandes y con mayor rendimiento económico del país, ocupa el puesto número 22 entre las empresas que mas empleados tiene en el país, revista semana, edición 1304 del 30 de abril al 07 de mayo de 2007.

El Tiempo, bogota 19 de septiembre 2008, Colanta regalará 100 mil litros de leche en barrios pobres de Bogotá y Medellín. Estos sucesos y la apertura que se tiene en los mercados internacionales, junto con la infraestructura que maneja en cuanto a plantas de procesamiento de las mas modernas en diferentes zonas del país, flota de vehículos para el transporte, las redes de distribución a nivel nacional, que la coloca como la primera productora y vendedora de leche en Colombia, y con una excelente estabilidad financiera, www.colanta.com.co/. Esto motivo al investigador a realizar un estudio y buscar estrategias para colocar los productos lácteos en el mercado internacional.

\section{Pensamiento estratégico como eje de cambio en mercado internacional}

Rubinstein, (1979). Argumenta que el pensamiento estratégico es el proceso de reflejo en la conciencia del hombre de la esencia de las cosas, de los vínculos y relaciones regulares entre los objetos o fenómenos de la realidad.

Según Martínez, (1998). Es la habilidad a mejorar y desarrollar, tramitando información, aperturándose a la toma de decisiones y al desenlace de interrogantes producto de situaciones al interactuar en su entorno.

Para los autores com Loehle, (1996;) y Wells (1998). El pensamiento estratégico pretende 
reducir la incertidumbre, minimizar los riesgos y maximizar las oportunidades a través de un conjunto de múltiples procedimientos de análisis y aprendizaje.

Como lo manifiesta el autor, el pensamiento estratégico es la base o el pilar de los procesos administrativos inherentes a su naturaleza organizacional, de igual forma parte de planes estratégicos para la toma de decisiones, a la vez le permite a la organización cumplir sus objetivos alcanzando metas, sin desviar la razón social de la empresa, y permitiéndole estar a la vanguardia de la competitividad.

\section{Pensar estratégicamente para ser competitivo}

Mintzberg (1998), Es una forma de pensar de manera responsable relacionada con la capacidad de emitir buenos juicios, mientras para Delphi. (2004) Es un proceso intelectual que, en forma decidida, regulada y autorregulada, busca llegar a un juicio razonable.

No obstante, Kurland (1995), Está relacionado con la razón, la honestidad intelectual y la amplitud mental en contraposición a lo emocional, a la pereza intelectual y a la estrechez mental.

Como lo manifiesta Kurland y apoyando su teoría, la persona responsable de la gestión organizacional debe cumplir el propósito planteado en la filosofía de gestión, apegado también a los planes estratégicos que guían las acciones para mantenerse competitiva en el mercado global.

\section{Estrategias pilar de gestión del negocio}

En las empresas, las decisiones acerca de que enfoque de negocios se deben adoptar y que nuevas medidas se deben iniciar, involucran a los ejecutivos, a los jefes de unidades, a los jefes de las principales áreas funcionales entre ellas fabricación, mercadotecnia, ventas, finan- zas, recursos humanos, entre otros, se tiene en cuenta a todo el personal para la formulación de estrategias que implican hacer elecciones y tomar decisiones de la organización, porque estas constituyen el modelo de negocios para producir una buena rentabilidad y buenos resultados de negocios. Conociendo lo que se busca con las estrategias vamos a conocer la definición de algunos autores expertos en la materia.

Según Thompson y Strickland (2004), estrategia consiste en los esfuerzos competitivos y los enfoques de negocio que los administradores utilizan para satisfacer a los clientes, competir exitosamente y alcanzar los objetivos de la organización. Las estrategias de la compañía conciernen al como: cómo lograr el crecimiento del negocio, como satisfacer a los clientes, como superar la competencia de los rivales, como responder a las condiciones cambiantes del mercado, como administrar cada parte funcional del negocio y desarrollar las capacidades organizacionales necesarias, como lograr los objetivos estratégicos y financieros.

Según Hill y Jones (2004), estrategia es la acción que los gerentes toman para alcanzar una o más de las metas de la organización. Para la mayoría de las organizaciones, si no es que para todas, una meta dominante es alcanzar un desempeño superior al de los competidores. La estrategia es el resultado de un proceso formal y de planeación y que el papel más importante en este corresponde a la alta dirección.

Según Munich y García (2004), el termino estrategia se deriva del griego estrategias, este quiere decir general, militarmente, se refiere a la manera de elegir aquellas acciones más adecuadas con la determinación de encausar los esfuerzos y derrotar al enemigo. Estos autores indican literalmente el significado al «arte de dirigir y coordinar las acciones, y de hacer una cosa para alcanzar el objetivo». 
Llevando el término al área administrativa, se puede decir que las estrategias son cursos de acción, los cuales indican la dirección, el empleo de los recursos y esfuerzos de la empresa, a manera de lograr los objetivos en las condiciones más ventajosas. Como lo manifiestan, las estrategias son la base fundamental y prioritaria de una organización, sin estas las organizaciones no pasarían de ser locales, sectoriales, no serian competitivas, permanecerían en un estado cataléptico.

Por lo expresado anteriormente, es necesario buscar estrategias que permitan demostrar cómo evoluciona la economía global, para que las compañías con capacidad de realizar transacciones internacionales, no se concentren únicamente en su país de origen, hay razones para internacionalizar las operaciones de una empresa como son: la sobrevivencia en lo económico, el crecimiento en lo estructural, tecnológico, personal y la diversificación en las diferentes regiones como en sus productos.

Este estudio permite identificar las estrategias más adecuadas para las empresas lácteas en el manejo del producto, precio, plaza, promoción, las 4P y la otra variable que va implícita dentro de este proceso la cual es la investigación, manejando en forma eficiente esta tarea se verá como las organizaciones van a tener un repunte en todos los campos viéndose reflejados en una parte importante del mercado.

\section{Marketing herramienta de proyección de bienes y servicios}

En los actuales momentos, dada las exigencias de los consumidores, las organizaciones deben llevar a cabo constantemente estudios y análisis de mercado para conocer las necesidades y deseos de éstos, con los productos que se fabriquen o comercialicen.

Stanton, Etzel, Walter, (2005), manifiesta al respecto que, la mercadotecnia o marketing es un sistema total de actividades de negocios diseñados para planear, fijar precios, promocionar y distribuir productos y servicios que satisfagan las necesidades a los mercados objetivos para lograr la metas organizacionales, donde el sistema de actividades de negocios debe orientarse hacia el consumidor reconociendo y/o satisfaciendo de modo efectivo las necesidades del cliente.

Kotler y otro (2003). Comenta al respecto que marketing es, la entrega de satisfacción a los clientes obteniendo una utilidad.

Schiffman y otro (2005) exponen sobre el concepto de marketing que, para alcanzar el éxito, una compañía debe determinar cuáles son las necesidades y los deseos de los mercados meta específicos y cumplir las satisfacciones deseadas mejor que sus competidores.

El concepto de marketing se basa en la premisa de que el mercadólogo debería producir lo que se vende, en lugar de tratar de vender lo que ya ha producido. Estos conceptos incluyen los siguientes aspectos:

1. Un enfoque de las necesidades del cliente, para que la empresa pueda diferenciar sus productos o servicios de la oferta de la competencia.

2. La integración de todas las actividades de la empresa, incluso la producción para satisfacer las necesidades.

3. Producir para satisfacer una necesidad o un deseo y no vender lo que está producido.

Los autores coinciden en que, el marketing es una técnica que, contribuye directamente a alcanzar los objetivos de las organizaciones, donde todas las personas de negocios, independiente de la especialización o área de responsabilidad, deben familiarizarse con esta tecnología, además el marketing no es solo el trabajo de las personas de un departamento de mercadotecnia, es parte de todas las personas de la empresa. 


\section{Estrategia de marketing}

Ferrell, Hartline y Lucas (2002), manifiesta que la estrategia de marketing de una organización se diseña para integrar los esfuerzos encaminados a lograr los objetivos de comercialización. Consiste en elegir uno o más mercados meta y luego desarrollar una mezcla de Marketing (producto, precio, promoción y distribución) que satisfaga las necesidades y deseos de los integrantes del mercado meta.

En líneas generales, las empresas específicamente las del sector lácteo se plantea mediante planes el modo en que obtendrá una ventaja competitiva haciendo benchmarking para tomar las mejores prácticas y mejorar las del competidor, por ello sus productos deben de ser de mejor calidad que lo que ofrecen los competidores, los precios deben ser congruentes con su grado de calidad, sus métodos de distribución deben ser lo más eficaces posibles, y sus promociones tienen que comunicarse en forma más adecuada a los clientes que la empresa se ha fijado como objetivo. También es importante que la compañía procure que estas ventajas resulten sostenibles.

\section{Marketing internacional una vía para el desarrollo económico binacional del sector lácteo}

Conociendo los conceptos de marketing, ahora se plantearan los conceptos de Marketing Internacional que es la variable que se va a estudiar en esta investigación para poder planificar, desarrollar y plantear las estrategias que se buscan al finalizar este trabajo. García-Sordo (2004), el concepto de marketing internacional se utiliza para referirse al proceso de comercializar productos y servicios en diferentes países.

Por consiguiente, Cateora (2006), manifiesta que es el desempeño de las actividades comerciales diseñadas para planificar, asignar precios, promover y dirigir el flujo de los bienes y servicios de una compañía a los consumidores o usuarios de más de un país con el fin de obtener ganancias.
Analizando los conceptos de marketing internacional se aprecia que los autores van encaminados a los mismos objetivos, como son buscar una planificación para tener intercambios que satisfagan las necesidades o deseos de los usuarios en los mercados externos, en este sentido se reviste de importancia para el mercado de lácteos Colombiano y Venezolano donde buscan alcanzar niveles de excelencia y productividad, a partir del abastecimiento de ellos en la sociedad, siendo estos los catalizadores de calidad, empleando para tal efecto estrategias de vanguardia, a fin de lograr su permanencia en el mercado.

Braidot, (1995), una empresa que decide incursionar en el mercado internacional debe considerar en primer lugar, en qué medida debe adaptar su producto y en general toda su mezcla de marketing, es decir la política de precios, de comunicación, de distribución, etc. A las condiciones y características del mercado de destino.

Hill y Jones, (2004), manifiesta que las empresas que buscan una estrategia internacional intentan crear valor mediante la transferencia de capacidades y productos valiosos a mercados extranjeros donde los competidores locales carecen de ellos. La mayoría de las empresas internacionales han creado valor a través de la transferencia de ofertas de productos diferenciados que han desarrollado en su país natal a los mercados extranjeros.

Por lo expresado anteriormente, el marketing internacional da las pautas para que las empresas exploren en otros mercados, es el caso de las empresas colombianas de lácteos que cumplen su meta de comercializar en el exterior, como lo está haciendo Alpina s.a, la cual ha extendido sus mercados por América-Latina con unos resultados satisfactorios. Hoy se encuentra en Venezuela donde empezó como exportador y hoy hace parte de la industria venezolana, porque tiene su empresa produciendo y distribuyendo 
en el mercado local, su meta es mejorar la posición en el mercado nacional si las políticas gubernamentales no siguen produciendo pánico a los inversionistas con la idea de nacionalizar las empresas de los países con diferente forma de pensar o de gobierno, el momento es optimo para tener relaciones comerciales en el área de la leche y sus derivados con Venezuela por la escasez de estos productos.

Con este tipo de productos varias empresas han incursionado en el campo internacional con buenos resultados, a Venezuela y Colombia han ingresado empresas como Nestlé, multinacional Suiza con la leche, complementada con sus derivados, mostrando a través de los años que su mercado ha ido en crecimiento reflejado en los anaqueles de los supermercados como en su estructura física. Este mismo caso sucedió con Parmalat, empresa Italiana ingreso primero a Venezuela con buenos resultados, luego a Colombia, con dificultades en su ingreso ya que este país es líder en empresas de leche, a pesar de esto se ha mantenido haciendo cambios en su política de ventas, presentación, como en la calidad de sus productos. Lo manifestado anteriormente a juicio del investigador por trabajar en esa área esa área.

Venezuela en este momento es un mercado potencial por la desatención del empresario tanto privado como público en el sector ganadero, han provocado incertidumbre en la industria de los productos lácteos en el país, por la escasez de la leche, por las diferentes políticas del gobierno, esta falencia se convierte en una oportunidad para las compañías que desean ingresar a este mercado.

Estos ejemplos sirven para tomar puntos de referencia de estas empresas y conocer cuál ha sido sus estrategias, saber las fortalezas, debilidades, así de esta manera hacer un estu- dio estructurado para encaminar los productos deseosos de ingresar a el mercado venezolano, estos alimentos deben de tener las mismas condiciones en cuanto a calidad y precio o mejorando lo anterior, para poder tener ventajas al momento de encontrarse en los anaqueles.

\section{Conclusiones}

La presente investigación tuvo como propósito analizar el pensamiento estratégico como base del marketing internacional en el sector lácteo. En función de dar respuesta a la proyección de los mercados internacional se presentan las conclusiones relevantes, derivadas del cumplimiento de factores investigativo.

Al analizar la situación actual de las empresas de productos lácteos en Colombia y Venezuela, se concluyó que manejan la logística adecuada para satisfacer la demanda de sus clientes y cuentan con proveedores de leche fijos.

De igual manera, se expresa que para los colombianos el factor económico internacional no representa una limitación en el funcionamiento de las empresas lácteas en Venezuela, por lo que, existen alianzas estratégicas para la adquisición de divisas destinadas a la compra de materia prima y la elaboración de los productos lácteos, sin embargo pudiesen en ocasiones establecerse restricciones generado de las políticas del país fronterizo en este caso Venezuela, y su inserción, a fin de abastecer estos productos de primera necesidad en la sociedad.

En cuanto al pensamiento estratégico mejoran y desarrollar, la información, aperturándose a la toma de decisiones y al desenlace de interrogantes, razonamiento que pretende reducir la incertidumbre, minimizar los riesgos y maximizar las oportunidades a través de situaciones al interactuar en su entorno. 
Al Identificar la percepción del mercado Venezolano sobre los productos lácteos Colombianos se concluyo que para los venezolanos las empresas de lácteos colombianas evalúan las necesidades del consumidor Venezolano antes de ubicar sus productos en este mercado.

Las estrategias de mercado de las empresas de lácteos colombianas, resaltan los sitios específicos donde conseguir los productos, así mismo, se mantiene un stop adecuado de los productos lácteos en los puntos de venta, para tener un manejo extenso y adecuado de los productos lácteos colombianos, se debe contar con distribuidores. De igual forma, las empresas de productos lácteos colombianas y venezolanas aplican estrategias de postventa, las mismas sirven para diferenciarse de los competidores.

\section{Recomendaciones}

En función de los resultados obtenidos en la presente investigación, se formulan las siguientes recomendaciones:

Es de suma importancia realizar estudios de mercadeo para detectar aquellos factores que originan las debilidades, buscando de esta manera mitigarlas y reforzar aquellas fortalezas en los mercados internacionales.

Rediseñar las estrategias de marketing internacional, aplicando estrategias de pensamiento estratégico que le permitan conseguir los objetivos deseados.

Se recomienda establecer y aplicar un Plan Estratégico de Mercadeo que facilite la penetración como la permanencia al mercado vene- zolano los productos lácteos colombianos, para lograr de esta forma una manera más ordenada y planificada que contribuya al incremento de los márgenes de rentabilidad.

Se invita a estar atento, prevenido los constantes cambios en los niveles económicos y cambiarios del país, considerando los niveles de inflación que repercuten en la estabilidad financiera de la empresa.

Se sugiere que los productos lácteos colombianos sean colocados manejando la distribución selectiva a través de representantes, contactando a socios comérciales que distribuyan este tipo de productos, utilizando herramientas como Internet, directorios del país en cuestión.

Contratar el asesoramiento o entes especializados en la materia para mitigar las debilidades presentadas en los canales de distribución, de este modo ser más eficientes y eficaces en la distribución de los productos lácteos colombianos, contribuyendo al incremento de la utilidad de los mismos.

Una vez iniciado el proceso de importación, se sugiere controlar y evaluar continuamente la efectividad de las estrategias de mercadeo y tácticas aplicadas, a través del monitoreo del mercado, permitiendo evaluar la gestión de los distribuidores.

De igual modo se sugiere extender este estudio hacia otras empresas, pues les permitirá a los gerentes mercadeo o de otras líneas visualizar el comportamiento del mismo y poder tomar acciones estratégicas a un corto plazo en función de mejoras continuas en cuanto a la relación con el cliente. 


\section{Referencias}

García-Sordo, Juan. (2007). Marketing Internacional. Mexico: Editorial Mc Graw-Hill Interamericana .

Cateora, Philip y Graham, John. (2006), Marketing Internacional. México: Editorial Mc Graw-Hill Interamericana .

ETzel, Walker. (2004). Fundamentos de Marketing, México: Editorial Mc Graw-Hill.

Kotler (2006). Dirección de Marketing, Madrid: Prentice-Hall.

Kotler y Amstrons. (2000). Fundamentos de Mercadotecnia, México: Prentice-Hall.

Schiffman (2005). Comportamiento del Consumidor, México: Prentice-Hall.

Thompson y Strickland (2004). Administración Estratégica, México: Mc Graw-Hill.

Hill y Jones (2005). Administración Estratégica, México: Mc Graw-Hill.

Ferrell, Hartline y Lucas (2002). Estrategia de Marketing, México: Internacional Thompson editores.

Fisher y Espejo (2002). Mercadotecnia, México: Mc Graw-Hill.

Pride y Ferrell (2003). Marketing: Conceptos y Estrategias, México: Mc Graw-Hill.

Serna (2003). Gerencia Estratégica, Bogota: 3R Editores.

Thompson (2004). Administración Estratégica. México: Editorial Mc Graw- Hill.

Lamb Charles, Hair Joseph y McDaniel Car (2002). Marketing. Mexico: International

Thomson editors s.a. Sexta Edicion.

Hiebing y Cooper, (2005). Como Preparar el Exitoso Plan de Mercadotecnia,

México: Mc Graw-Hill Interamerica. 